\title{
Three-dimensional transvaginal ultrasonographic and power Doppler features of adenomyosis : Are they related to symptoms?
}

\author{
Original \\ Article
}

\author{
Reda Abdul-aziz Ahmad \\ Department of Obstetrics and Gynecology, Faculty of Medicine, Zagazig University, Zagazig, \\ Egypt
}

\begin{abstract}
Aim of the work: To investigate the relationship between specific 2D and 3D ultrasound and power Doppler features of adenomyosis and the commonly associated adenomyosis symptoms in absence of other pelvic pathologies.

Patients and Methods: This is a prospective observational study done at the Obstetrics and Gynecology Ultrasound Unit, Zagazig University Hospitals. This study was carried out from September 2015 to February 2017. It included all premenopausal women complaining of abnormal uterine bleeding, pain, subfertility and others. Cases without ultrasound detected pathology were collected as a control group (273), while cases having only adenomyosis ultrasonographic features by 2D-transvaginal sonography were collected as adenomyosis group (148). 2D-TVS myometrial assessment was performed for all women. 3D-TVS was then performed for full visualization of the junctional zone. VOCAL was used to calculate the Doppler indices of the myometrium.

Results: The number of ultrasound findings was independent predictor for occurrence of menstrual disorders and chronic pelvic pain. Junctional zone irregularity was a predictor for heavy menstruation and chronic pelvic pain, increased uterine volume and asymmetry were significantly correlated with chronic pelvic pain, while subfertility might be predictable with fan shaped shadowing and myometrial cysts. All myometrial Doppler indices were significantly higher in adenomyotic cases compared to control group. Increased myometrial VI and VFI were strongly correlated with the occurrence of heavy menstruation and inter-menstrual bleeding.

Conclusion: We found a relationship between certain ultrasound features of adenomyosis and some of associated symptoms. This relationship can help in understanding the pathophysiology of symptoms related to adenomyosis.
\end{abstract}

Key Words: Adenomyosis, Doppler, symptoms, three-dimensional, ultrasound.

Received: 12 January 2019, Accepted: 27 January 2019

Corresponding Author: Reda Abdul-aziz Ahmad, Department of Obstetrics \& Gynecology, Faculty of Medicine, Zagazig University, Zagazig, Egypt, Tel.: 00201129585885, E-mail: Redaaahmad71@gmail.com.

ISSN: 2090-7265, May 2019, Vol.9, No.2

\section{INTRODUCTION}

Adenomyosis is defined as myometrial invasion by endometrial glands and stroma. It shows a wide variance in estimated prevalence from 5 up to $70 \%$ with an average of $20-30 \%{ }^{[1,2]}$. While, premenopausal women represent the usual population, it can affect young women and was even found in adolescents ${ }^{[3-6]}$.

Like its prevalence, the clinical presentation is also heterogeneous. While, $20-30 \%$ of cases may stay asymptomatic ${ }^{[7]}$, patients may complain of one or more complaint. Abnormal uterine bleeding (heavy menstrual bleeding and inter-menstrual bleeding) and/ or painful menstruation are the most frequent ${ }^{[8,}$, 3 . Dyspareunia and chronic pelvic pain come as less frequent complaints $^{[4,10]}$. Also, with the rising trend of delayed marriage and late seeking for first pregnancy till late thirties or more, adenomyosis was implicated to have negative impact on fertility and the success of IVF cycles $^{[11-13]}$.

Until recently, adenomyosis was a retrospective histologic diagnosis on removed uteri. Now, imaging techniques including MRI and 2D transvaginal ultrasound gave the chance for diagnosing adenomyosis with high sensitivity and specificity ${ }^{[14,15]}$. The advent of threedimensional ultrasound allowed quick and clear evaluation of junctional zone added to the value of Doppler study of blood flow distribution in differentiating adenomyosis from leiomyoma reducing the need for costly MRI ${ }^{[16]}$. In 2015, the Morphological Uterus Sonographic Assessment (MUSA) group proposed a statement for uniform reporting sonographic features of the myometrial lesions including $\operatorname{adenomyosis}^{[17]}$.

Some researchers tried to examine the relationship between adenomyosis and different symptoms including abnormal uterine bleeding, painful menstruation, chronic pelvic pain and subfertility. Most of these studies failed 
to establish a strong correlation between adenomyosis and these symptoms ${ }^{[18-22]}$. This in part was due to the fact of coexistence of other pelvic pathologies (e.g. myomas, endometriosis) in up to $80 \%$ of adenomyosis cases, making distinguishing the adenomyosis cause effect relationship difficult ${ }^{[23]}$.

The aim of this study was to investigate the relationship between specific 2D and 3D ultrasound and power Doppler features of adenomyosis and the commonly associated adenomyosis symptoms in absence of other pelvic pathologies. To the best of our knowledge, we are the first to examine the predictability of adenomyosis symptoms according to ultrasound features in absence of other known confounders.

\section{PATIENTS AND METHODS}

This prospective observational study was carried out at the Obstetrics and Gynecology Ultrasound Unit, Zagazig University Hospitals during the period from September 2015 to February 2017 after approval by the institutional review board of Zagazig University (ZU-IRB\#3314-15-8-2015). Premenopausal women complaining of abnormal uterine bleeding, pain, subfertility and/or others who were referred from the gynecology outpatient clinics to our unit for sonographic evaluation of pelvic organs were considered for the study after an informed oral consent.

Patients were then divided into 2 groups: Women without any detected ultrasound pathology (control group) and women who had at least one of the features typical of adenomyosis observed by 2D-TVS including myometrial antero-posterior asymmetry, fan-shaped shadowing, myometrial cysts, hyperechogenic islands, subendometrial lines and buds, irregular or ill-defined junctional zone (adenomyosis group). Women on hormonal treatments or medications that affect menstrual bleeding or those discovered to have sonographic features of endometriosis, fibroids, or other pelvic pathologies were excluded from the study.

The single main complaint was recorded including abnormal bleeding (heavy menstruation or inter-menstrual bleeding), pain (painful menstruation, dyspareunia, chronic pelvic pain) or subfertility. The amount of menstrual blood loss was evaluated using the pictorial blood loss assessment chart ${ }^{[24]}$. Heavy menstruation was diagnosed in patients with a score of $\geq 100$ points. Local gynecologic examination was performed to exclude cervical or vaginal pathology.

2D and 3D gray-scale and power Doppler ultrasound imaging were then carried out by one expert examiner with an experience of more than 10 years in gynecologic ultrasound, who was blinded to the patients' symptoms and to the initial ultrasonographic finding, using a $5-9 \mathrm{MHz}$ endovaginal probe with three-dimensional facility (Voluson E6, GE Medical Systems, Zipf, Austria). In women with regular cycles, examination was performed in the secretory phase. Pelvic organs were first assessed to exclude any extracorporeal or endometrial pathology, and any myometrial pathology other than adenomyosis, then the myometrium was assessed according to the terms and definitions of the Morphological Uterus Sonographic Assessment (MUSA) group ${ }^{[17]}$.

\section{D-TVS examination included:}

(1) uterine measurements: in a midsagittal plane of the uterus, longitudinal corporeal diameter ( $\mathrm{d} 1$; from the fundal serosal surface to the internal os), longest anteroposterior diameter ( $h$; height), anterior (a) and posterior ( $\mathrm{p}$ ) myometrial wall thickness (from uterine serosa to outer endometrial border, including the junctional zone (JZ) not the endometrium, in each wall) and endometrial thickness (e) were measured. The probe was then rotated $90^{\circ}$ and the longest transverse diameter $(\mathrm{d} 2)$ was measured in the transverse plane (Fig. 1, 2).

The ratio between both myometrial walls thicknesses was calculated (thicker/thinner uterine wall ratio). Uterine walls were considered asymmetric when this ratio was $>1.5$. The actual antero-posterior myometrial diameter $(\mathrm{d} 3)$ was calculated by subtracting endometrial thickness from the longest antero-posterior diameter. Myometrial volume was then calculated [volume $\left.\left(\mathrm{cm}^{3}\right)=\mathrm{d} 1 \mathrm{xd} 2 \mathrm{xd} 3 \times 0.523\right]$.

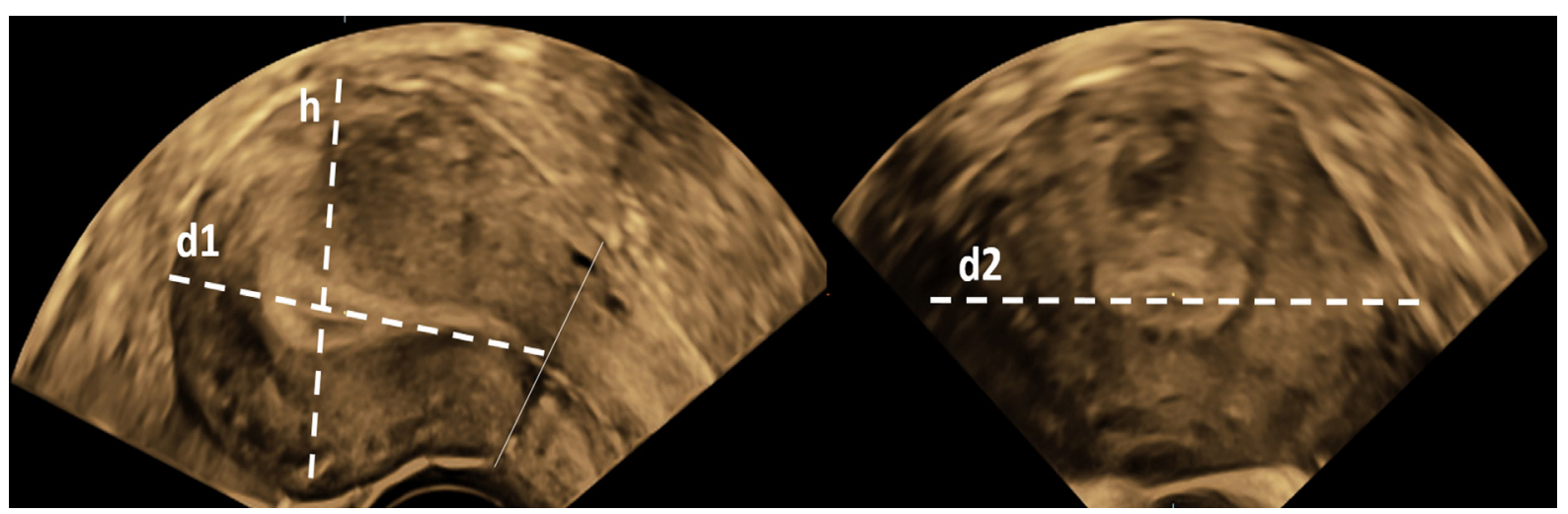

Fig. 1: Measurement of longitudinal (d1), transverse (d2) and antero-posterior (h) corporeal diameters. To the left: A midsagittal plane of the uterus, longitudinal corporeal diameter ( $\mathrm{d} 1$; from the fundal serosal surface to the internal os) and the longest anteroposterior diameter (h), were measured. To the right: The probe was then rotated $90^{\circ}$ and the longest transverse diameter $(\mathrm{d} 2)$ was measured in the transverse plane. 


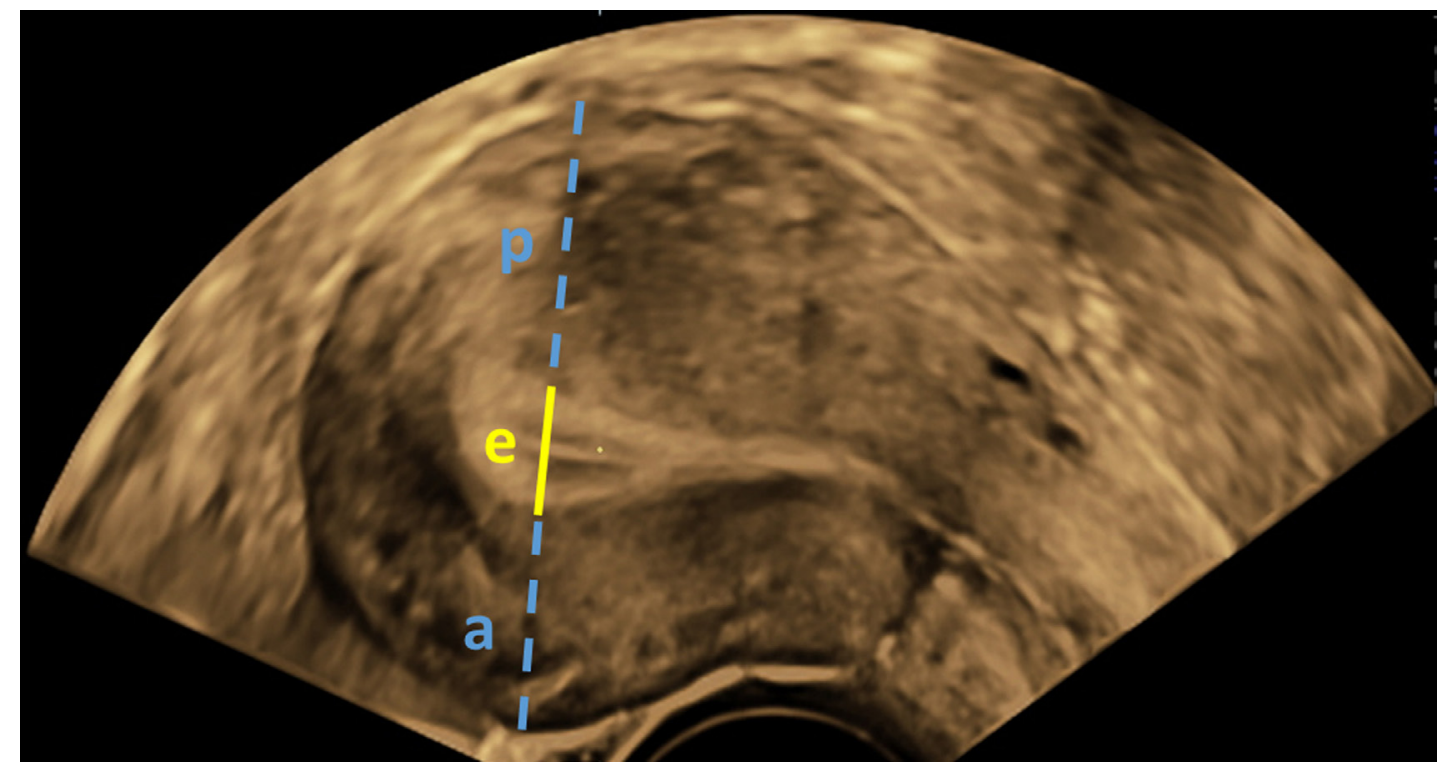

Fig. 2: Measurement of anterior (a) and posterior (p) myometrial wall thickness (from uterine serosa to outer endometrial border, including the junctional zone (JZ) not the endometrium, in each wall) and endometrial thickness (e).

(2) Myometrial echogenicity was described as homogenous or heterogeneous. Reason for heterogeneity was recorded as one or more of the following: Fanshaped shadowing (Fig 3a), myometrial cysts (with their number, mean diameter of the largest cyst and location: in inner half or outer half of the myometrium) (Fig 3b), hyperechogenic islands (with determination of their extent: diffuse; $\geq 50 \%$ of whole myometrium or localized; $<50 \%$ of whole myometrium, and their penetration: $<50 \%$ or $\geq 50 \%$ of myometrial wall thickness) (Fig 3c). (3) Presence or absence of hyperechogenic subendometrial lines or buds (Fig 3d). The so-called 'question-mark sign' was not used as a diagnostic sign for adenomyosis, because it can also be seen in posterior compartment deep infiltrating endometriosis ${ }^{[25]}$. Moreover, we didn't include it in the studied ultrasonographic features of adenomyosis as it occurs secondary to asymmetric myometrial thickening.

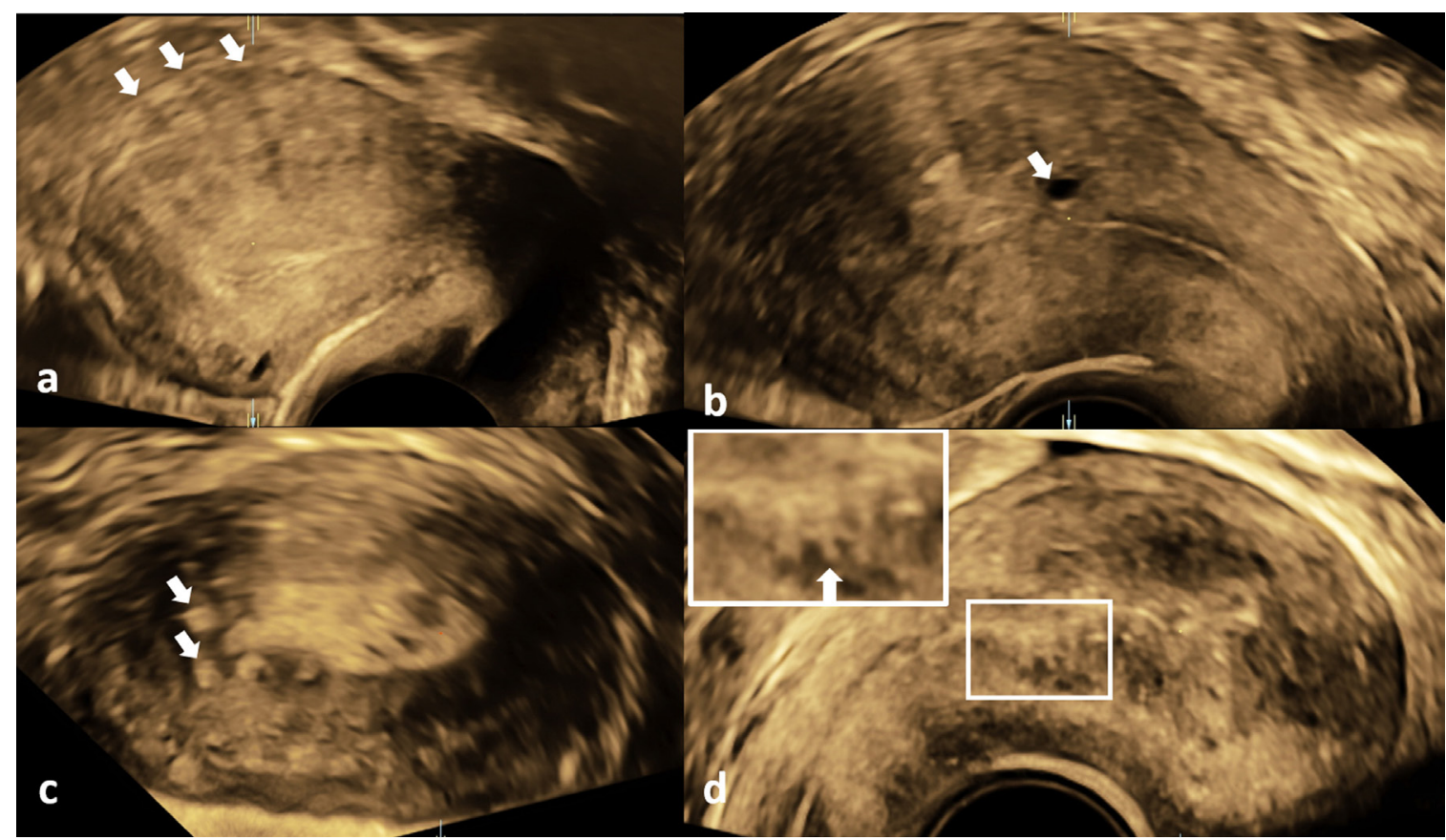

Fig. 3: Fan-shaped shadowing (hypoechogenic ray-like lines) (a), myometrial cyst (rounded anechoic lesion within the myometrium) (b), hyperechogenic islands (hyperechogenic areas within the myometrium) (c), hyperechogenic subendometrial lines (hyperechogenic lines projecting from the endometrium towards the myometrium and disrupting the JZ) (d). 
Three-dimensional volume acquisition was then performed with volume box and sweep angle adjusted to include the whole serosal surface of the uterine corpus (Quality: high 1). Two volumes were acquired, one by VCI (Volume Contrast Imaging) mode (with $2 \mathrm{~mm}$ slice thickness) and the other by glass body mode acquired with power Doppler applied on the whole myometrium. Doppler settings were fixed for all patients (frequency, mid; pulse repetition frequency, $0.6 \mathrm{kHz}$; gain, -6 ; wall motion filter, 'low 2'). VCI acquired volumes were manipulated in multiplanar view to obtain the coronal plane of uterine corpus, for full visualization and study of the junctional zone. In some cases, the endometrial stripe was not straight due to asymmetric thickening of uterine walls with bulging myometrium, leading to indentation of the endometrial line (question-mark sign). In those cases, we applied

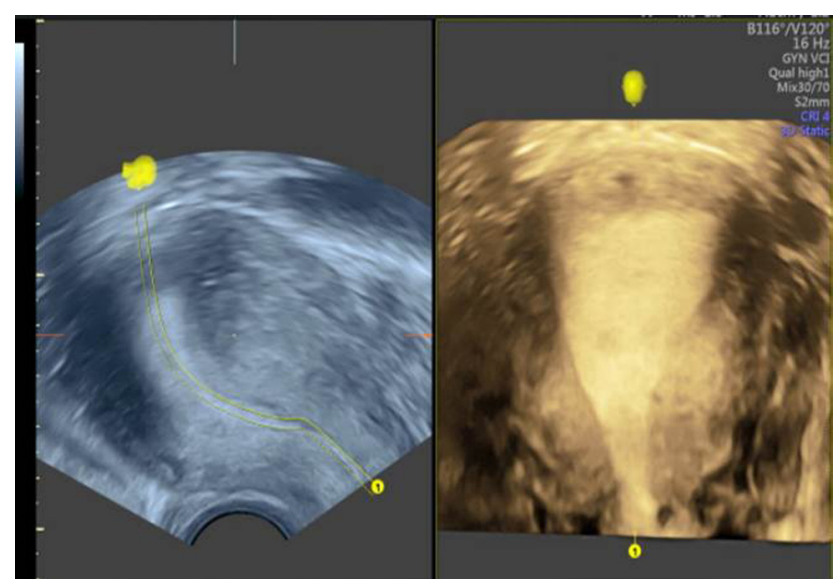

Fig. 4: Coronal plane reconstruction by OmniView polyline mode. Applied when the endometrial stripe was not straight (due to asymmetric thickening of uterine walls with bulging myometrium, leading to indentation of the endometrial line; question-mark sign).

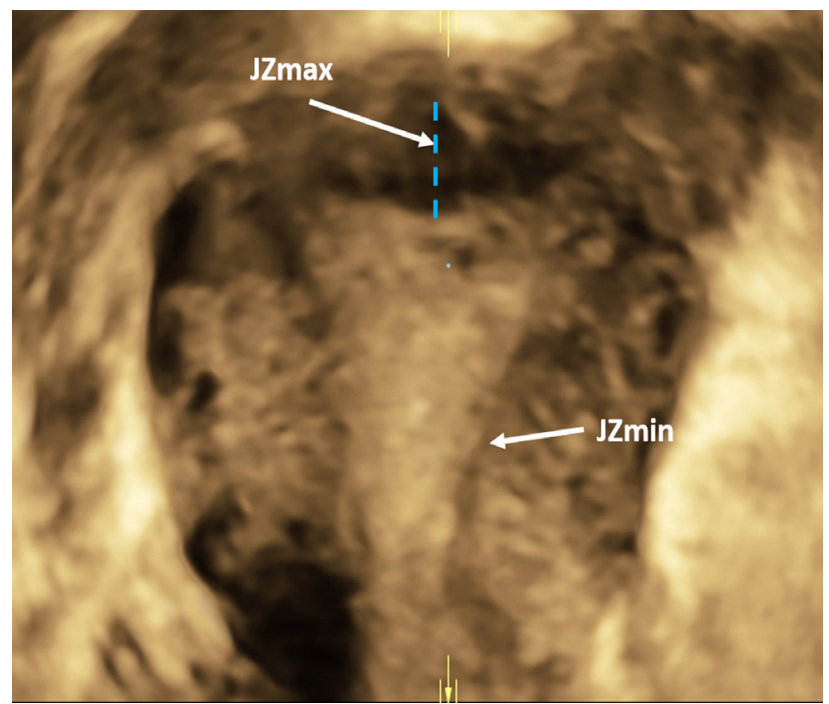

Fig. 5: The junctional zone (JZ) magnitude of irregularity, calculated by subtracting the minimum from the maximum thickness of JZ (JZmax - JZmin).
Omni View polyline mode to reconstruct the coronal plane (Fig 4). JZ was described as being regular, irregular or ill-defined. In irregular JZ, the magnitude of irregularity was calculated by subtracting the minimum from the maximum thickness of JZ (JZdif=JZmax - JZmin) (Fig 5). Junctional zone irregularity was considered marked when JZdif was $\geq 4 \mathrm{~mm}^{[26]}$. Virtual organ computer-aided analysis (VOCALTM) was used to calculate the vascularity index (VI), the flow index (FI) and the vascularization flow index (VFI) of the myometrium (using the histogram), by tracing the uterine corpus only, avoiding inclusion of the parametrial vessels and the uterine artery (Fig 6). The endometrium couldn't be excluded from the tracing due to the irregularity of the junctional zone present in all cases included in the study, making accurate demarcation of the endometrium impossible.
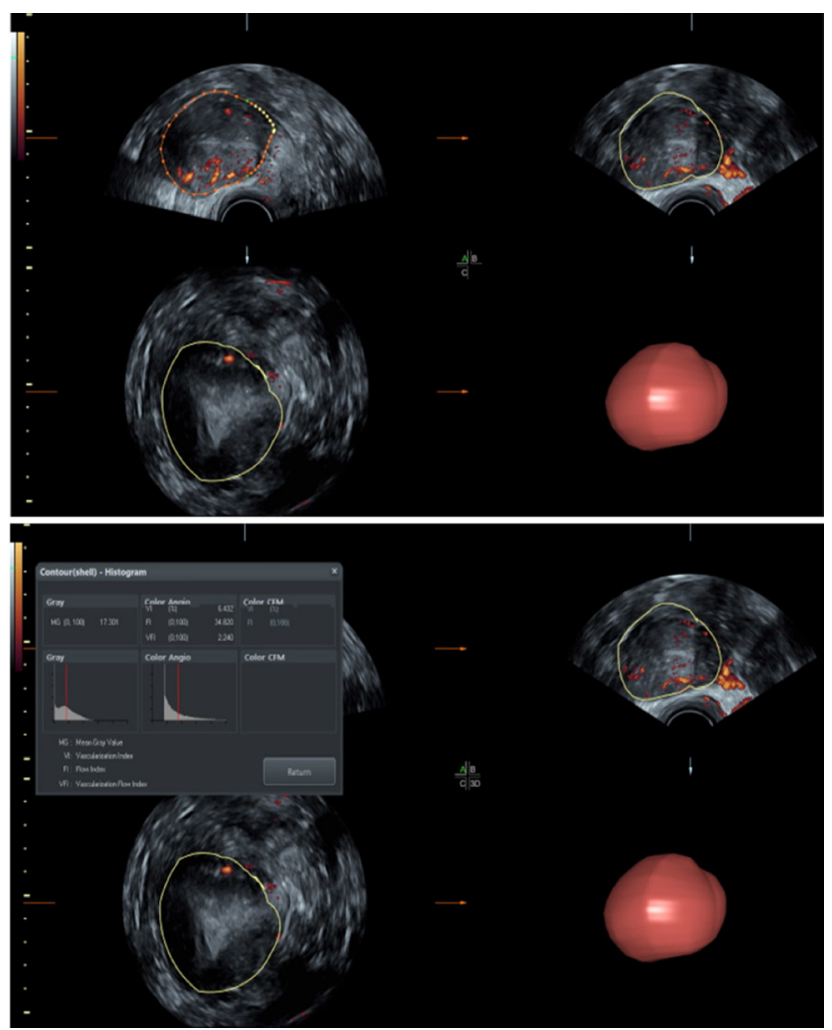

Fig. 6: Virtual organ computer-aided analysis (VOCALTM) with calculation of the vascularity index (VI), the flow index (FI) and the vascularization flow index (VFI) using histogram.

For study cases who have undergone hysterectomy (32 patients), the histopathological reports were reviewed as a confirmation or exclusion of adenomyosis.

Statistical analysis was performed using the following software products: SPSSC version 21 [IBM $\odot$ Corp., Armonk, NY]. Shapiro-Wilk test was used to examine the numerical data for normality of distribution. Skewed data were presented as median and interquartile range (IQR). Normally distributed data were presented as mean 
\pm standard deviation (SD). Categorical data were presented as number and percentage (\%). Independent t-test was done for comparison of demographic data, uterine wall asymmetry, and Doppler study data between the case and control groups.

Sensitivity, specificity, negative (NPV) and positive (PPV) predictive values, positive and negative likelihood ratios (LR) and accuracy of each studied ultrasound feature suggesting adenomyosis for hysterectomized cases were calculated depending on the histopathologic results.

Multi-nominal logistic regression model was used to test every ultrasound feature as an independent predictor for each symptom in the adenomyosis cases. Goodness of fit of model was confirmed using Pearson and Deviance tests, Pseudo R square was $83.2 \%$ (Cox and Snell), 85.8\% (Negelkerk). All the ultrasound features suggesting adenomyosis were adjusted, including myometrial volume, uterine wall asymmetry, junctional zone (JZ) irregularity, JZ difference, fan-shaped shadowing, presence and the site of myometrial cysts, hyperechogenic islands, subendometrial buds and Doppler indices of myometrial vasculature. $P$ value $<0.05$ was considered statistically significant.

\section{RESULTS}

During the study period, 1245 patients were reviewed in the gynecologic outpatient clinics and referred for ultrasound evaluation. Six hundred and two (48.4\%) cases showed a variety of pelvic pathologies other than adenomyosis, these patients were not included in the study. One or more of the proposed ultrasound features of adenomyosis were detected in 370 patients (29.7\%), of which $58.4 \%$ (216 out of 370) were excluded due to concomitant presence of other lesions (e.g. fibroids, endometrial polyps and endometriomas). The remaining 148 patients $(11.9 \%$ of total population, $40 \%$ of adenomyotic cases) showed only ultrasonographic features of adenomyosis and were included in this study. The remaining 273 examined patients $(21.9 \%)$ were sonographically free from any detectable pelvic pathology and were included in the study as a control group. (Figure 7)

Women in the adenomyosis group were older $(44.3 \pm 8.2)$ with tendency toward higher parity as compared to control group ( $P$-value 0.001), BMI was comparable between both groups (Table 1).

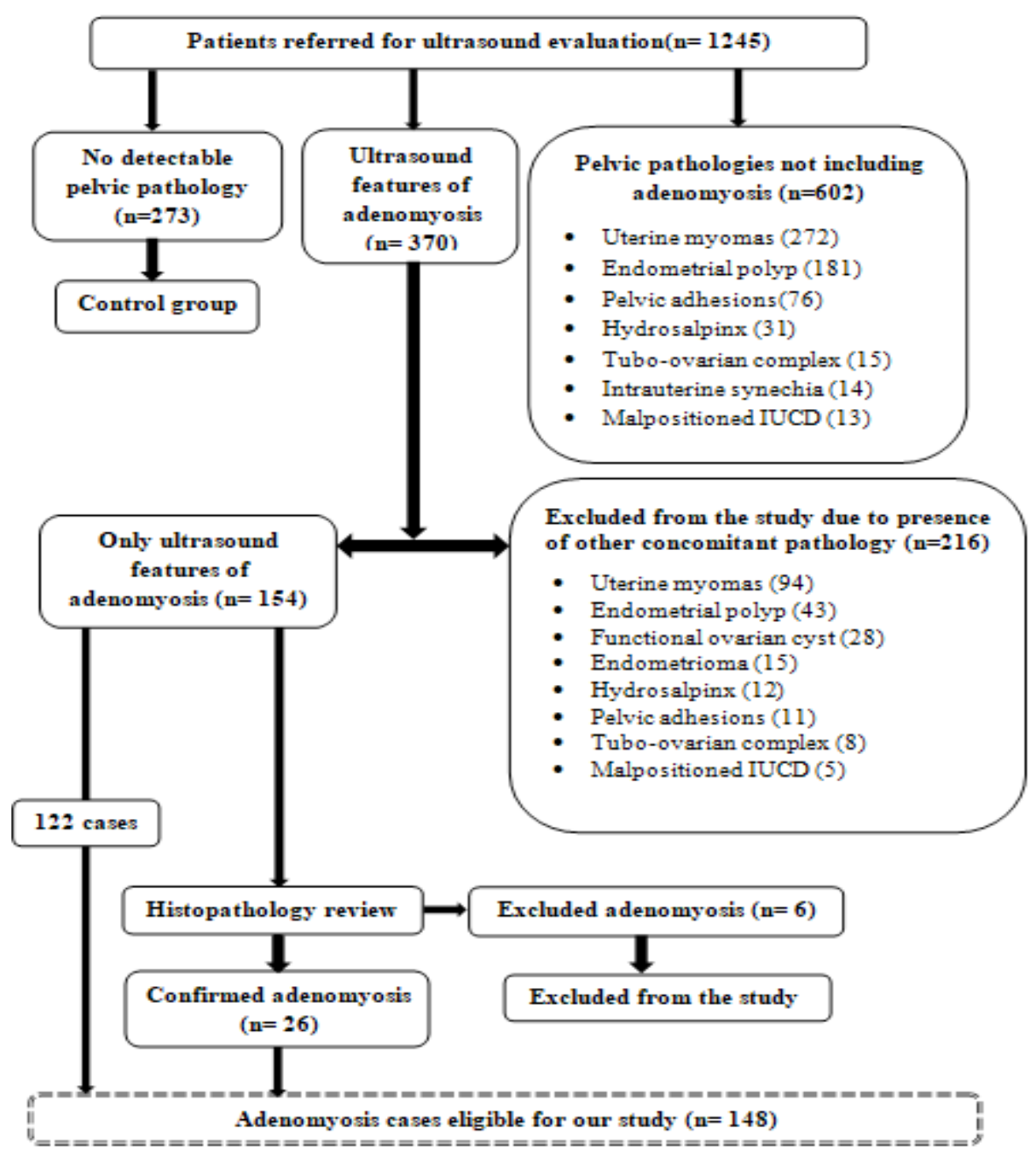

Fig, 7: Flow diagram 
Table 1: Patient demographic data and frequency of their main symptoms.

\begin{tabular}{|c|c|c|c|}
\hline Variable & $\begin{array}{l}\text { Adenomyosis group } \\
\qquad(\mathrm{n}=148)\end{array}$ & $\begin{array}{l}\text { Control group } \\
\qquad(\mathrm{n}=273)\end{array}$ & $P$ value \\
\hline Age (years, mean $\pm \mathrm{SD}$ ) & $44.3 \pm 8.2$ & $39.6 \pm 7.3$ & 0.001 \\
\hline Less than 30 years & $8(5.4 \%)$ & $19(7 \%)$ & - \\
\hline $30-<40$ years & $30(20.3 \%)$ & $74(27.1 \%)$ & - \\
\hline$\geq 40$ years & $110(74.3 \%)$ & $180(65.9 \%)$ & - \\
\hline BMI & $26.7 \pm 2.3$ & $25.9 \pm 2.8$ & 0.231 \\
\hline Parity & $3(3-4)$ & $2(0-3)$ & 0.000 \\
\hline Nullipara & $8(5.4 \%)$ & $78(28.6 \%)$ & - \\
\hline Para1,2 & $22(14.9 \%)$ & $102(37.4 \%)$ & - \\
\hline Para $\geq 3$ & $118(79.7 \%)$ & $93(34 \%)$ & - \\
\hline \multicolumn{4}{|l|}{ Main complaint } \\
\hline Heavy menstruation (n (\%)) & $40(27.8 \%)$ & $43(15.8 \%)$ & 0.007 \\
\hline Inter-menstrual bleeding (n (\%)) & $33(22.3 \%)$ & $27(9.8 \%)$ & 0.001 \\
\hline Chronic pelvic pain (n (\%)) & $24(16.2 \%)$ & $48(17.6 \%)$ & 0.722 \\
\hline Painful menstruation (n (\%)) & $10(6.8 \%)$ & $45(16.5 \%)$ & 0.004 \\
\hline Subfertility (n (\%)) & $10(6.8 \%)$ & $57(20.9 \%)$ & 0.000 \\
\hline Dyspareunia (n (\%)) & $6(4.1 \%)$ & $19(7 \%)$ & 0.183 \\
\hline Other reasons (n (\%)) & $25(16.9 \%)$ & $34(12.4 \%)$ & 0.254 \\
\hline
\end{tabular}

SD standard deviation, BMI body mass index

Data are presented as mean \pm standard deviation, number (percentage) and median (interquartile range) as appropriate.

The main symptoms of frequency for the adenomyotic cases were heavy menstruation $(27.8 \%)$, inter-menstrual bleeding $(22.3 \%)$, chronic pelvic pain $(16.2 \%)$, dysmenorrhea $(6.8 \%)$, subfertility $(6.8 \%)$ and dyspareunia (4.1\%). A considerable percentage of cases $(16.9 \%)$ with ultrasound features of adenomyosis, were either asymptomatic cases came for checkup or with unrelated complaints (e.g. loin pain, iliac pain, abnormal vaginal discharge.....etc.). However, symptoms were significantly more frequent in the adenomyosis group than the control group except for chronic pelvic pain, dyspareunia and other reasons (Table 1). Out of the 15 ultrasound features, junctional zone irregularity, heterogeneous myometrial echogenicity, hyperechogenic islands and subendometrial echogenic buds were fixed findings in almost all adenomyotic cases. The other features were detected in varying percentages (Figure 8). The myometrial volume, uterine wall asymmetry and 3D power Doppler indices were significantly higher in adenomyotic group compared to control group (Table 2). 


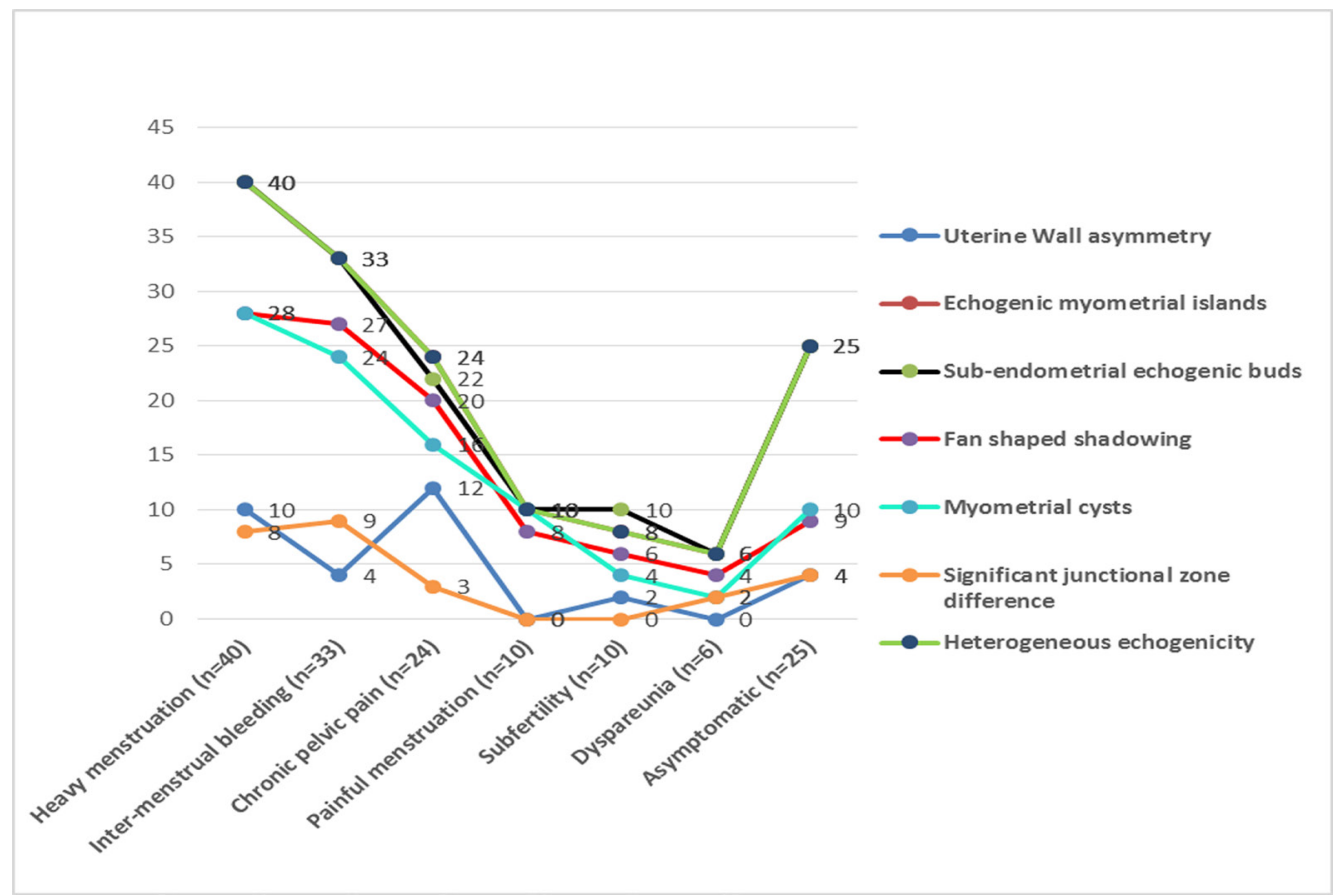

Fig. 8: Frequency of US features related to each symptom

Table 2: Frequency of ultrasound features of adenomyosis

\begin{tabular}{|c|c|c|c|}
\hline Parameter & $\begin{array}{l}\text { Adenomyosis group } \\
\qquad(\mathrm{n}=148)\end{array}$ & $\begin{array}{l}\text { Control group } \\
(\mathrm{n}=273)\end{array}$ & $P$ value \\
\hline Myometrial volume (mean $\pm \mathrm{SD}$ ) & $103.8 \pm 60.4$ & $76.3 \pm 12.4$ & 0.001 \\
\hline Uterine walls asymmetry [n (\%)] & $32(21.6 \%)$ & 0 & - \\
\hline Thicker / thinner uterine wall ratio (mean $\pm \mathrm{SD}$ ) & $1.4 \pm 0.6$ & $1.05 \pm 0.12$ & 0.001 \\
\hline Junctional zone (JZ) Irregularity [n (\%)] & $148(100 \%)$ & - & - \\
\hline Less than $50 \%$ & $16(10.8 \%)$ & - & - \\
\hline More than $50 \%$ & $84(56.8 \%)$ & - & - \\
\hline Ill-defined JZ & $48(32.4 \%)$ & - & - \\
\hline $\mathrm{JZ}$ dif. $\geq 4 \mathrm{~mm}$ & $26(17.6 \%)$ & - & - \\
\hline Heterogenous myometrial echogenicity [n (\%)] & $146(98.6 \%)$ & - & - \\
\hline Fan-shaped shadowing [n (\%)] & $102(68.9 \%)$ & - & - \\
\hline Myometrial cysts $[\mathrm{n}(\%)]$ & $94(63.5 \%)$ & - & - \\
\hline Inner half of uterine wall & $62(41.9 \%)$ & - & - \\
\hline Both inner and outer & $32(21.6 \%)$ & - & - \\
\hline Number of myometrial cysts (median-IQR) & $3(2-6)$ & - & - \\
\hline Largest cyst mean diameter $(\mathrm{mm})($ mean $\pm \mathrm{SD})$ & $2.5 \pm 2.2$ & - & - \\
\hline Echogenic islands [n (\%)] & $146(98.6 \%)$ & - & - \\
\hline Sub-endometrial buds[n (\%)] & $146(98.6 \%)$ & - & - \\
\hline $3 \mathrm{D}$ power Doppler indices of the myometrium $($ mean $\pm \mathrm{SD})$ & & - & - \\
\hline VI $(\%)$ & $9.6 \pm 5.7$ & $4.7 \pm 1.2$ & 0.000 \\
\hline FI $(0-100)$ & $36.4 \pm 4.1$ & $30.6 \pm 1.4$ & 0.001 \\
\hline VFI $(0-100)$ & $3.3 \pm 2.1$ & $1.2 \pm 0.04$ & 0.001 \\
\hline No. of ultrasound features per patient & $6(5-7)$ & - & - \\
\hline
\end{tabular}

SD standard deviation, JZ dif. junctional zone difference, VI vascularization index, FI flow index, VFI vascularization flow index. Data are presented as mean \pm standard deviation and number (percentage) as appropriate. 
After review of the histopathologic reports of cases who had hysterectomy (32 cases), the diagnosis of adenomyosis was confirmed in 26 cases (overall sensitivity $81.3 \%$ ). Most of ultrasonographic features showed high sensitivity (57.7- 92.3\%), specificity $(83.3-50 \%)$ and accuracy $(62.5-87.5 \%)$ in predicting the presence of adenomyosis (Table 3 ).

Regression analysis showed significant correlation between number of positive ultrasound features of adenomyosis and presence of heavy menstruation, inter-menstrual bleeding and chronic pelvic pain. Increased myometrial vascularization and vascularization flow Doppler indices were found as independent predictors of both heavy menstrual and inter-menstrual bleeding. Mean uterine volume was positively correlated to the occurrence of chronic pelvic pain, while it was negatively correlated to subfertility. Fan shaped shadowing was positively correlated to chronic pelvic pain and subfertility. Extensive junctional zone disruption and uterine walls asymmetry were found as significant independent predictors for chronic pelvic pain; while myometrial cysts were also significant for subfertility. None of the 15 features showed a significant correlation to painful menstruation and dyspareunia (Table 4).

Table 3: Accuracy of individual ultrasonographic adenomyosis features in cases with post-hysterectomy histopathologic examination ( $\mathrm{n}=32)$

\begin{tabular}{lccccccc}
\hline Ultrasound feature & Sens. $\%$ & Spec. $\%$ & PPV & NPV & LR+ & LR- & Accuracy $\%$ \\
\hline Uterine wall asymmetry & 57.7 & 83.3 & 93.8 & 31.2 & 3.46 & 0.52 & 62.5 \\
Heterogeneous echogenicity & 84.6 & 50.0 & 88.0 & 42.9 & 1.69 & 0.31 & 78.1 \\
JZ dif. $\geq$ 4mm & 73.1 & 83.3 & 95.0 & 41.7 & 4.38 & 0.20 & 75.0 \\
JZ Irregularity & 88.5 & 66.7 & 92.0 & 57.1 & 2.66 & 0.17 & 84.4 \\
Fan-shaped shadowing & 76.9 & 66.7 & 90.9 & 40.0 & 2.31 & 0.35 & 75.0 \\
Myometrial cysts & 80.0 & 83.3 & 95.5 & 50.0 & 4.79 & 0.24 & 81.2 \\
Echogenic islands & 92.3 & 66.7 & 92.3 & 66.7 & 2.77 & 0.12 & 87.5 \\
Subendometrial buds & 81.2 & 83.3 & 95.7 & 55.6 & 4.86 & 0.23 & 84.4 \\
\hline
\end{tabular}

Table 4: Multi-nominal logistic regression model showing different ultrasound features as significant independent predictors of common symptoms of patients with adenomyosis.

\begin{tabular}{lcccccc}
\hline Symptom & Covariate & B & SE & OR & $95 \%$ CI & $P$ \\
\hline & Number of positive findings & 1.913 & 0.680 & 6.775 & $1.788-25.675$ & 0.005 \\
Heavy menstruation & JZ irregularity (more than 50\%) & 3.196 & 1.426 & 0.041 & $0.003-0.669$ & 0.025 \\
& Myometrial VI & 2.561 & 0.839 & 2.943 & $2.501-12.036$ & 0.036 \\
& Myometrial VFI & 0.347 & 0.537 & 1.407 & $1.039-1.906$ & 0.027 \\
& Number of positive findings & 1.888 & 0.730 & 6.606 & $1.581-27.600$ & 0.010 \\
Inter-menstrual bleeding & Myometrial VI & 0.666 & 0.299 & 0.514 & $0.286-0.924$ & 0.026 \\
& Myometrial VFI & 1.578 & 0.794 & 4.844 & $1.022-22.954$ & 0.047 \\
& Number of positive findings & 2.166 & 0.817 & 8.724 & $1.760-43.249$ & 0.008 \\
Chronic pelvic pain & Mean uterine volume & 0.061 & 0.024 & 0.941 & $0.897-0.987$ & 0.012 \\
& JZ irregularity (more than 50\%) & 3.583 & 1.546 & 0.028 & $0.001-0.575$ & 0.020 \\
Painful menstruation & Fan shadowing & 3.494 & 1.492 & 0.030 & $0.002-0.566$ & 0.019 \\
& Uterine wall asymmetry & 3.196 & 1.901 & 0.041 & $0.001-1.369$ & 0.043 \\
Subfertility & & & None & & & $0.934-34.294$ \\
& Myometrial volume & -0.238 & 0.074 & 0.788 & 0.001 \\
Dyspareunia & Fan shadowing & 8.406 & 3.161 & 0.030 & $0.002-0.566$ & 0.008 \\
\hline & Myometrial cysts & 5.973 & 2.666 & 0.003 & $1.730-0.473$ & 0.025
\end{tabular}

- 95\% CI, 95\% confidence interval; B, regression coefficient; OR, odds ratio; SE, standard error.

- Deviance goodness of fit test was insignificant for the model.

- Pseudo R square was $83.2 \%$ (Cox and Snell), $85.8 \%$ (Negelkerk). 


\section{DISCUSSION}

In this study, we focused on searching for significant association between individual ultrasound features and specific complaints. While, a considerable sector of cases may be asymptomatic, the majority of cases with adenomyosis complain of a variety of symptoms. These symptoms may be produced or aggravated by the type, location and degree of spread of that pathology in the myometrium beside the common concomitant pathologies like endometriosis and myomas ${ }^{[18,27]}$.

Adenomyosis features were detected in $29.7 \%$ of all symptomatic cases seen during the period of the study, this prevalence raises the need for considering the possibility of adenomyosis during ultrasound evaluation, especially in premenopausal patients ${ }^{[8]}$. Going with the findings of previous studies ${ }^{[7,28]}$, heavy menstrual bleeding was the most frequent complaint, followed by inter-menstrual bleeding (27.8\% and $22.3 \%$, respectively). Chronic pelvic pain came after abnormal uterine bleeding (16.2\%). Interestingly, painful menstruation was less frequent symptom in comparison to previous studies, where it was the most common complaint $(6.8 \% \text { vs } 15-80 \%)^{[1,3,5,10,29]}$. The frequency of occurrence of these symptoms was much different for the control group where the subfertility, chronic pelvic pain and painful menstruation were the most frequent complaints (Table 1). For precise studying of the relationship between ultrasound features of adenomyosis and symptoms, all cases with concomitant pathology that may modulate the type and degree of symptoms were excluded. Only patients whose symptoms could be apparently attributed to adenomyosis alone were included, despite inability to confirm absence of other unknown causes for these symptoms.

Junctional zone (JZ) irregularity (with varying degrees), heterogeneous myometrial echogenicity, echogenic islands and subendometrial echogenic buds were fixed findings in almost all cases ; all of these features have shown high sensitivity after review of histopathology for hysterectomy cases (81-92\%). This makes us acknowledge them as good screening ultrasound features for adenomyosis. Of notice, 25 patients $(16.9 \%)$ had these features and were asymptomatic. Other findings including fan-shaped shadowing, myometrial cysts and uterine wall asymmetry were detected less frequently (Table 2). In our study, echogenic islands, subendometrial echogenic buds, JZ irregularity and myometrial cysts showed the highest accuracy (Table 3). Similar results were found by other authors $^{[5,26]}$.

Among different theories, in an interesting explanation, Leyendecker et al. discussed the pathophysiology of adenomyosis based on defective tissue injury and repair mechanisms, starting by migration of basal endometrial glands through the iatrogenically or auto-traumatized junctional zone (archimetrium) followed by progressive growth and spread via hyperperistalsis ${ }^{[30]}$. These glands would be the precursors of micro- and macro-cysts and according to its distribution and interaction with the surrounding hyperplastic myometrium will produce almost all other ultrasound findings

We examined all listed ultrasound features (Table 2) to find significant independent predictors for each symptom. Despite few ultrasound findings showed a correlation strong enough to be counted as an independent predictor for specific symptoms (according to the analysis of our cases), it was clear that the number of detected ultrasound features was the strongest independent predictor of such symptoms. This was applicable for heavy menstruation, inter-menstrual bleeding and chronic pelvic pain. It seems logic that the abundant ultrasound features mirror the extensive affection of the uterus leading to considerable congestion explaining the heavy menstruation and chronic pelvic pain. Also for both symptoms, partial/complete loss of JZ was an independent predictor. The marked destruction of junctional zone mirrors the diffuse spread of adenomyosis, smooth muscle hyperplasia and increased vascularity and congestion of the uterus. Also, the hyperperistalsis or convulsive dysperistalsis of the heavily invaded or completely lost JZ will increase the menstrual loss and provokes chronic pelvic pain ${ }^{[31]}$.

Chronic pelvic pain was correlated with the highest number of ultrasound features. Beside the previously mentioned junctional zone irregularity, fan shaped shadowing, uterine wall volume and asymmetry were also significant predictors. All these features will be associated with congestion and dysperistalsis which would precipitate such chronic complaint ${ }^{[31]}$.

For inter-menstrual bleeding, the increased number of findings which indicates the extent of uterine affection was a significant predictor. This type of bleeding could be attributed to the associated endometrial defects and cracking. In such cases, these defects were documented during hysteroscopic evaluation in some studies ${ }^{[32]}$. This cracked endometrium will be prone for bleeding away from the time of normal shedding and with the dysrhythmic JZ activity, recurrent cracking will lead to such bleeding.

None of the features were significant as predictor for painful menstruation and dyspareunia. Pain was attributed by some authors to increased prostaglandin production in adenomyotic nodules in comparison to normal myometrium ${ }^{[33]}$. Naftalin et al. ${ }^{[19]}$ found that the severity of pain during menstruation increases in correlation with the number of ultrasound findings, this positive correlation was not significant in our work. Presence of many myometrial cysts with the retained blood inside may cause pain on pressure on the uterus during intercourse, causing dyspareunia. However, the small number of cases with both symptoms is a point of weakness for their results.

Subfertility was negatively correlated with the uterine volume. Of course, the small uterus in such cases is a logic finding rather than a predictor, as with parity the uterus 
becomes larger. Fan shaped shadowing and presence of myometrial cysts were the independent predictors for subfertility. Both mirror the scatter of the micro/macrocystic lesions and adenomyotic foci that may induce abnormal peristalsis and affects implantation ${ }^{[9,34]}$.

The value of 3D Doppler indices with adenomyosis is still questioned ${ }^{[17]}$. Doppler study included vascularization index, flow index and vascularization flow index. Till now, there are no standardized cutoff values for comparison of these indices. All Doppler indices were found significantly higher in adenomyotic cases compared to the control group. VI and VFI were found as significant predictors for heavy menstruation and inter-menstrual bleeding. This may reflect the role of myometrial congestion in both conditions. Similar values of these indices were found in other studies ${ }^{[35]}$.

Really, both the ultrasound features and symptoms of adenomyosis are reflection of a continuum of this progressive and heterogeneous pathology. In our trial, to find a specific correlation between these ultrasound features of adenomyosis and the nature of associated symptoms and in the light of the previous findings, we found that patients with many ultrasound features were prone to be more symptomatic, especially for bleeding disorders and chronic pelvic pain. Patients with uterine asymmetry and large volume are predicted to have chronic pelvic pain. Lastly, patients with more myometrial cysts and fan shadowing may be more prone to fertility problems.

In conclusion, the possibility of adenomyosis should be put in mind during the evaluation of gynecologic complains, especially in premenopausal women. Threedimensional ultrasound and Doppler evaluation gives a detailed description of different features of adenomyosis. We found a relationship between certain ultrasound features of adenomyosis and some of the associated symptoms. We believe that this relationship can help in understanding the pathophysiology of symptoms related to adenomyosis.

Further research may be required to establish cutoff values for normal and abnormal 3D Doppler indices. Validation of a classification system of severity of adenomyosis according to symptoms and ultrasound features needs further studies.

\section{ACKNOWLEDGMENTS}

My sincere thanks to Dr. Hytham Atia (Zagazig University) for help with manuscript preparation. I thank Dr. Somayya Sadek (Zagazig University) for assistance in 3D ultrasound assessment of my cases.

\section{CONFLICT OF INTEREST}

There are no conflicts of interest.

\section{REFERENCES}

1. Parazzini F, Mais V, Cipriani S, et al. Determinants of adenomyosis in women who underwent hysterectomy for benign gynecological conditions: Results from a prospective multicentric study in Italy. Eur J Obstet Gynecol Reprod Biol 2009; 143: 103-106.

2. Parazzini F, Mais V, Cipriani S, et al. Determinants of adenomyosis in women who underwent hysterectomy for benign gynecological conditions: Results from a prospective multicentric study in Italy. Eur J Obstet Gynecol Reprod Biol 2009; 143: 103-106.

3. Bergholt T, Eriksen L, Berendt N, et al. Prevalence and risk factors of adenomyosis at hysterectomy for adenomyosis . Hum Reprod 2001; 16: 2418-2421.

4. Li X, Liu X, Guo SW. Clinical profiles of 710 premenopausal women with adenomyosis who underwent hysterectomy. J Obstet Gynaecol Res 2014; 40: 485-494.

5. Taran FA, Stewart EA, Brucker S. Adenomyosis: Epidemiology, risk factors, clinical phenotype and surgical and interventional alternatives to hysterectomy. Geburtshilfe Frauenheilkd 2013; 73: 924-931.

6. Pinzauti S, Lazzeri L, Tosti C, et al. Transvaginal sonographic features of diffuse adenomyosis in 18-30-year-old nulligravid women without endometriosis: Association with symptoms. Ultrasound Obstet Gynecol 2015; 46: 730-736.

7. Ryan GL. An Unusual Cause of Adolescent Dysmenorrhea. 2006; 108: 1017-1022.

8. Peric H, Fraser IS. The symptomatology of adenomyosis. Best Pract Res Clin Obstet Gynaecol 2006; 20: 547-555.

9. Basak S, Saha A. Adenomyosis: still largely underdiagnosed. J Obstet Gynaecol 2009; 29: 533-535.

10. Kissler S, Zangos S, Kohl J, et al. Duration of dysmenorrhoea and extent of adenomyosis visualised by magnetic resonance imaging. Eur J Obstet Gynecol Reprod Biol 2008; 137: 204-209.

11. Boeer B, Wallwiener M, Rom J, et al. Differences in the clinical phenotype of adenomyosis and leiomyomas: A retrospective, questionnaire-based study. Arch Gynecol Obstet 2014; 289: 1235-1239.

12. Maheshwari A, Gurunath S, Fatima $\mathrm{F}$, et al. Adenomyosis and subfertility: a systematic review 
of prevalence, diagnosis, treatment and fertility outcomes. 2012; 18: 374-392.

13. Vercellini P, Consonni D, Dridi D, et al. Uterine adenomyosis and in vitro fertilization outcome: a systematic review and meta-analysis. 2014; 29: 964-977.

14. Thalluri V, Tremellen KP. Ultrasound diagnosed adenomyosis has a negative impact on successful implantation following GnRH antagonist IVF treatment. 2012; 27: 3487-3492.

15. Campo S, Campo V, Benagiano G. Adenomyosis and infertility. Reprod Biomed Online 2012; 24: 35-46.

16. Graziano A, Monte GLO, Piva I, et al. Diagnostic findings in adenomyosis: a pictorial review on the major concerns. Eur Rev Med Pharmacol Sci 2015; 19: 1146-1154.

17. Sharma K, Bora MK, Venkatesh BP, et al. Role of 3D ultrasound and doppler in differentiating clinically suspected cases of leiomyoma and adenomyosis of uterus. J Clin Diagnostic Res 2015; 9: QC08-QC12.

18. Van Den Bosch T, Dueholm M, Leone FPG, et al. Terms, definitions and measurements to describe sonographic features of myometrium and uterine masses: A consensus opinion from the Morphological Uterus Sonographic Assessment (MUSA) group. Ultrasound Obstet Gynecol 2015; 46: 284-298.

19. Naftalin J, Hoo W, Pateman K, et al. Is adenomyosis associated with menorrhagia? Hum Reprod 2014; 29: 473-479.

20. Naftalin J, Hoo W, Nunes $\mathrm{N}$, et al. Association between ultrasound features of adenomyosis and severity of menstrual pain. Ultrasound Obstet Gynecol 2016; 47: 779-783.

21. Levgur M, Abadi MA, Tucker A. Adenomyosis: Symptoms, histology, and pregnancy terminations. Obstet Gynecol 2000; 95: 688-691.

22. Sammour A, Pirwany I, Usubutun A, et al. Correlations between extent and spread of adenomyosis and clinical symptoms. Gynecol Obstet Invest 2002; 54: 213-216.

23. Parker JD, Leondires M, Sinaii N, et al. Persistence of dysmenorrhea and nonmenstrual pain after optimal endometriosis surgery may indicate adenomyosis. Fertil Steril 2006; 86: 711-715.

24. Garcia L, Isaacson K. Adenomyosis : Review of the Literature. J Minim Invasive Gynecol; 18: 428-437.
25. HIGHAM JM, O'BRIEN PMS, SHAW RW. Assessment of menstrual blood loss using a pictorial chart. BJOG An Int $\mathrm{J}$ Obstet Gynaecol 1990; 97: 734-739.

26. Donato N Di, Bertoldo V, Montanari G, et al. A SIMPLE SONOGRAPHIC SIGN ASSOCIATED TO THE PRESENCE OF ADENOMYOSIS. Ultrasound Obs Gynecol 2015; 46: 126-127.

27. Exacoustos C, Brienza L, Di Giovanni A, et al. Adenomyosis: Three-dimensional sonographic findings of the junctional zone and correlation with histology. Ultrasound Obstet Gynecol 2011; 37: 471-479.

28. Genc M, Genc B, Cengiz H. Adenomyosis and accompanying gynecological pathologies. Arch Gynecol Obstet 2015; 291: 877-881.

29. Bird CC, McElin TW, Manalo-estrella P. The elusive adenomyosis of the uterus. Am J Obs Gynecol 1972; 112: 583-593.

30. Bergeron C, Amant F, Ferenczy A. Pathology and physiopathology of adenomyosis. Best Pract Res Clin Obstet Gynaecol 2006; 20: 511-521.

31. Leyendecker G, Wildt L, Mall G. The pathophysiology of endometriosis and adenomyosis: Tissue injury and repair. Arch Gynecol Obstet 2009; 280: 529-538.

32. Brosens JJ, Barker FG, DeSouza NM. Myometrial zonal differentiation and uterine junctional zone hyperplasia in the non-pregnant uterus. Hum Reprod Update 1998; 4: 496-502.

33. Molinas CR, Campo R. Office hysteroscopy and adenomyosis. Best Pract Res Clin Obstet Gynaecol 2006; 20: 557-567.

34. Struble J, Reid S, Bedaiwy MA. Adenomyosis; A Clinical Review of a Challenging Gynecologic Condition. J Minim Invasive Gynecol. Epub ahead of print 2015. DOI: 10.1016/j.jmig.2015.09.018.

35. Pontis A, Alterio MND, Pirarba $\mathrm{S}$, et al. Adenomyosis : a systematic review of medical treatment Adenomyosis : a systematic review of medical treatment. 2016; 3590: 0-5.

36. Elkattan E, Kamel R, Elghazaly $\mathrm{H}$, et al. Can Three-dimensional (3D) power Doppler and uterine artery Doppler differentiate between fibroids and adenomyomas? Middle East Fertil Soc J 2016; 21: 46-51. 